\title{
INTERSECTION THEORY FOR LAGRANGIAN IMMERSIONS
}

\author{
MANABU AKAHO
}

\begin{abstract}
We introduce Floer homology for transversely intersecting Lagrangian immersions $L$ and $L^{\prime}$ in a symplectic manifold $(X, \omega)$. By using this homology, if $\pi_{2}(X, L)=0$ and $L^{\prime}$ is the image of $L$ under a Hamiltonian isotopy, then the number of the intersection points of $L$ and $L^{\prime}$ is bounded below by the sum of the $\mathbf{Z}_{2}$-betti numbers of $L$ (or rather, the manifold whose immersion is $L$ ) and a non-negative extra term coming from the self-intersections of $L$.
\end{abstract}

\section{Introduction}

Let $X$ be a symplectic manifold of dimension $2 n$ and $\omega$ its symplectic form. We call a map $\alpha: \tilde{L} \rightarrow X$ a Lagrangian immersion if and only if $\alpha$ is an immersion of an $n$-dimensional manifold $\tilde{L}$ into $X$ and the pull-back $\alpha^{*} \omega$ vanishes. A self-intersection point $x \in \alpha(\tilde{L})$ is called transverse if $\tilde{x} \in \alpha^{-1}(x)$ and $\tilde{x}^{\prime} \in$ $\alpha^{-1}(x), \tilde{x} \neq \tilde{x}^{\prime}$, have neighborhoods $U_{\tilde{x}} \subset \tilde{L}$ and $U_{\tilde{x}^{\prime}} \subset \tilde{L}$ respectively such that $\alpha\left(U_{\tilde{x}}\right)$ and $\alpha\left(U_{\tilde{x}^{\prime}}\right)$ are submanifolds intersecting transversally at $x$. If the number of the elements of $\alpha^{-1}(x)$ is two, then we call $x$ double. Similarly, for two Lagrangian immersions $\alpha_{0}: \tilde{L}_{0} \rightarrow X$ and $\alpha_{1}: \tilde{L}_{1} \rightarrow X$, an intersection point $x \in \alpha_{0}\left(\tilde{L}_{0}\right) \cap \alpha_{1}\left(\tilde{L}_{1}\right)$ is called transverse if $\tilde{x}_{0} \in \alpha_{0}^{-1}(x)$ and $\tilde{x}_{1} \in \alpha_{1}^{-1}(x)$ have neighborhoods $U_{\tilde{x}_{0}} \subset \tilde{L}_{0}$ and $U_{\tilde{x}_{1}} \subset \tilde{L}_{1}$ respectively such that $\alpha_{0}\left(U_{\tilde{x}_{0}}\right)$ and $\alpha_{1}\left(U_{\tilde{x}_{1}}\right)$ are submanifolds intersecting transversally at $x$. If the number of the elements of $\alpha_{0}^{-1}(x) \cup \alpha_{1}^{-1}(x)$ is two, then we call $x$ double. We will prove the following theorem:

Theorem 1.1. Let $X$ be a closed symplectic manifold and $\alpha: \tilde{L} \rightarrow X$ a Lagrangian immersion of a closed manifold $\tilde{L}$ whose self-intersection points are transverse and double. Suppose $\pi_{2}(X, \alpha(\tilde{L}))=0$. Let $\left\{\varphi_{t}\right\}_{0 \leq t \leq 1}$ be a Hamiltonian isotopy on $X$ such that the intersection points of $\alpha(\tilde{L})$ and $\left(\varphi_{1} \circ \alpha\right)(\tilde{L})$ are transverse and double. Then we have

$$
\sharp\left\{\alpha(\tilde{L}) \cap\left(\varphi_{1} \circ \alpha\right)(\tilde{L})\right\} \geq \operatorname{rank} H_{*}\left(\tilde{L}, \mathbf{Z}_{2}\right)+2 N,
$$

where $N$ is the number of the self-intersection points of $\alpha(\tilde{L})$.

The main purpose of this paper is to define Floer homology for Lagrangian immersions. Because we use immersions instead of embeddings, we have to introduce the appropriate path space as the domain of the symplectic action functional, and take care about the removal of boundary singularity with boundary

Received by the editors July 7, 2003. 
on Lagrangian immersions [8]. However, with the path space, almost all analytical results carry over as in the case of Lagrangian submanifolds. Theorem 1.1 follows from calculation of the homology for $\alpha(\tilde{L})$ and $\left(\varphi_{1} \circ \alpha\right)(\tilde{L})$.

\section{Analytical preliminaries}

Let $\alpha_{0}: \tilde{L}_{0} \rightarrow X$ and $\alpha_{1}: \tilde{L}_{1} \rightarrow X$ be Lagrangian immersions whose selfintersection points are transverse and double. Suppose that the intersection points of $\alpha_{0}\left(\tilde{L}_{0}\right)$ and $\alpha_{1}\left(\tilde{L}_{1}\right)$ are also transverse and double.

We denote by $I\left(\alpha_{0}, \alpha_{1}\right)$ the set $\left\{\left(\tilde{x}_{0}, \tilde{x}_{1}\right) \in \tilde{L}_{0} \times \tilde{L}_{1} \mid \alpha_{0}\left(\tilde{x}_{0}\right)=\alpha_{1}\left(\tilde{x}_{1}\right)\right\}$. For $\tilde{x}_{-}=\left(\tilde{x}_{-}^{0}, \tilde{x}_{-}^{1}\right)$ and $\tilde{x}_{+}=\left(\tilde{x}_{+}^{0}, \tilde{x}_{+}^{1}\right) \in I\left(\alpha_{0}, \alpha_{1}\right)$, we define $W^{\infty}\left(\tilde{x}_{-}, \tilde{x}_{+}\right)$to be the set of $\left(u, \tilde{u}_{0}, \tilde{u}_{1}\right)$, where $u \in C^{\infty}(\mathbf{R} \times[0,1], X)$ and $\tilde{u}_{i} \in C^{\infty}\left(\mathbf{R}, \tilde{L}_{i}\right)$ such that $\alpha_{i} \circ \tilde{u}_{i}(\tau)=u(\tau, i)$ and $\lim _{\tau \rightarrow \pm \infty} \tilde{u}_{i}(\tau)=\tilde{x}_{ \pm}^{i}$ for $i=0,1$. For $p k>2$, we denote by $W_{k}^{p}\left(\tilde{x}_{-}, \tilde{x}_{+}\right)$the completion of $W^{\infty}\left(\tilde{x}_{-}, \tilde{x}_{+}\right)$by the norm $\left\|\left(u, \tilde{u}_{0}, \tilde{u}_{1}\right)\right\|_{L_{k}^{p}}:=\|$ $u \|_{L_{k}^{p}}$, where $\|\cdot\|_{L_{k}^{p}}$ is the Sobolev $L_{k}^{p}$ norm, i.e., the $l$-th derivatives, for $l \leq k$, are $L^{p}$-integrable. Similarly, we define $W_{k}^{p}\left(S^{1} \times[0,1], X ; \alpha_{0}, \alpha_{1}\right)$ to be the $L_{k}^{p}$-completion of the set of $\left(u, \tilde{u}_{0}, \tilde{u}_{1}\right)$, where $u \in C^{\infty}\left(S^{1} \times[0,1], X\right)$ and $\tilde{u}_{i} \in C^{\infty}\left(S^{1}, \tilde{L}_{i}\right)$ such that $\alpha_{i} \circ \tilde{u}_{i}(\tau)=u(\tau, i)$ for $i=0,1$.

To construct coordinate charts on $W_{k}^{p}\left(\tilde{x}_{-}, \tilde{x}_{+}\right)$as a Banach manifold, we should introduce a family of metrics on $X$. Let $S_{0}:=\left\{p_{1}, \ldots, p_{m_{0}}\right\}$ be the set of the self-intersection points of $\alpha_{0}\left(\tilde{L}_{0}\right)$. We take small $\varepsilon>0$ such that closed $2 n$-dimensional balls $B_{2 \varepsilon}\left(p_{i}\right)$ in $X$, centered at $p_{i}$ with radius $2 \varepsilon$, do not intersect each other and $B_{2 \varepsilon}\left(p_{i}\right) \cap \alpha_{0}\left(\tilde{L}_{0}\right)$ are homeomorphic to two $n$-disks intersecting at $p_{i}$. For $p_{i} \in S_{0}$, there are two points $p_{i}^{u}$ and $p_{i}^{d}$ in $\alpha_{0}^{-1}\left(p_{i}\right)$. We denote by $B_{\varepsilon}^{u}\left(p_{i}\right)$ and $B_{\varepsilon}^{d}\left(p_{i}\right)$ the connected components of $\tilde{L}_{0} \cap \alpha_{0}^{-1}\left(B_{\varepsilon}\left(p_{i}\right)\right)$ containing $p_{i}^{u}$ and $p_{i}^{d}$ respectively. Similarly, let $S_{1}:=\left\{q_{1}, \ldots, q_{m_{1}}\right\}$ be the set of the self-intersection points of $\alpha_{1}\left(\tilde{L}_{1}\right)$ and let $B_{\varepsilon}^{u}\left(q_{i}\right)$ and $B_{\varepsilon}^{d}\left(q_{i}\right)$ be the connected components of $\tilde{L}_{1} \cap \alpha_{1}^{-1}\left(B_{\varepsilon}\left(q_{i}\right)\right)$ containing $q_{i}^{u}$ and $q_{i}^{d}$ respectively. Then we introduce a family of metrics $g^{(t, s)}$ on $X$ parameterized by $(t, s) \in[0,1] \times[-1,1]$ such that $\alpha_{0}\left(\tilde{L}_{0} \backslash \bigcup_{i=1}^{m_{0}} B_{\varepsilon}^{d}\left(p_{i}\right)\right), \alpha_{0}\left(\tilde{L}_{0} \backslash \bigcup_{i=1}^{m_{0}} B_{\varepsilon}^{u}\left(p_{i}\right)\right)$ and $\alpha_{0}\left(\tilde{L}_{0}\right) \backslash \bigcup_{i=1}^{m_{0}} B_{\varepsilon}\left(p_{i}\right)$ are totally geodesic with respect to $g^{(0,1)}, g^{(0,-1)}$ and $g^{(0, s)}$ respectively, and similarly $\alpha_{1}\left(\tilde{L}_{1} \backslash \bigcup_{i=1}^{m_{1}} B_{\varepsilon}^{d}\left(q_{i}\right)\right), \alpha_{1}\left(\tilde{L}_{1} \backslash \bigcup_{i=1}^{m_{1}} B_{\varepsilon}^{u}\left(q_{i}\right)\right)$ and $\alpha_{1}\left(\tilde{L}_{1}\right) \backslash \bigcup_{i=1}^{m_{1}} B_{\varepsilon}\left(q_{i}\right)$ are totally geodesic with respect to $g^{(1,1)}, g^{(1,-1)}$ and $g^{(1, s)}$ respectively. Next, we take a smooth function $s_{0}: \tilde{L}_{0} \rightarrow[-1,1]$ such that $s_{0}(x)=1, s_{0}(x)=-1$ and $s_{0}(x)=0$ for $x \in \bigcup_{i=1}^{m_{0}} B_{\varepsilon}^{u}\left(p_{i}\right), x \in \bigcup_{i=1}^{m_{0}} B_{\varepsilon}^{d}\left(p_{i}\right)$ and $x \in \tilde{L}_{0} \backslash \alpha_{0}^{-1}\left(\bigcup_{i=1}^{m_{0}} B_{2 \varepsilon}\left(p_{i}\right)\right)$ respectively. Similarly, $s_{1}: \tilde{L}_{1} \rightarrow[-1,1]$ is a smooth function such that $s_{1}(x)=$ $1, s_{1}(x)=-1$ and $s_{1}(x)=0$ for $x \in \bigcup_{i=1}^{m_{1}} B_{\varepsilon}^{u}\left(q_{i}\right), x \in \bigcup_{i=1}^{m_{1}} B_{\varepsilon}^{d}\left(q_{i}\right)$ and $x \in$ $\tilde{L}_{1} \backslash \alpha_{1}^{-1}\left(\bigcup_{i=1}^{m_{1}} B_{2 \varepsilon}\left(q_{i}\right)\right)$ respectively. For $\left(u, \tilde{u}_{0}, \tilde{u}_{1}\right) \in W_{k}^{p}\left(\tilde{x}_{-}, \tilde{x}_{+}\right)$, we choose an $L_{k}^{p}$ function $s_{u}: \mathbf{R} \times[0,1] \rightarrow[-1,1]$ such that the restriction on $\mathbf{R} \times\{0\}$ and $\mathbf{R} \times\{1\}$ is equal to $s_{0} \circ \tilde{u}_{0}$ and $s_{1} \circ \tilde{u}_{1}$ respectively and $s_{u}(\tau, t)=0$ for large $|\tau|$.

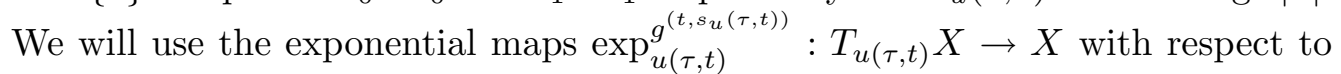
the metrics $g^{\left(t, s_{u}(\tau, t)\right)}$ to construct the coordinate charts. 
If $E$ is a smooth vector bundle over $X$ and $\left(u, \tilde{u}_{0}, \tilde{u}_{1}\right)$ is an element of $W_{k}^{p}\left(\tilde{x}_{-}, \tilde{x}_{+}\right)$, then the pull-back bundle $u^{*} E$ has the structure of an $L_{k}^{p}$-bundle. We can therefore define $L_{l}^{q}\left(u^{*} E\right)$ by the set of $L_{l}^{q}$-sections of $u^{*} E$, provided that $l \leq k$ and $l-2 / q \leq k-2 / p$. In particular, we are interested in the following Banach space for $\left(u, \tilde{u}_{0}, \tilde{u}_{1}\right) \in W_{k}^{p}\left(\tilde{x}_{-}, \tilde{x}_{+}\right)$

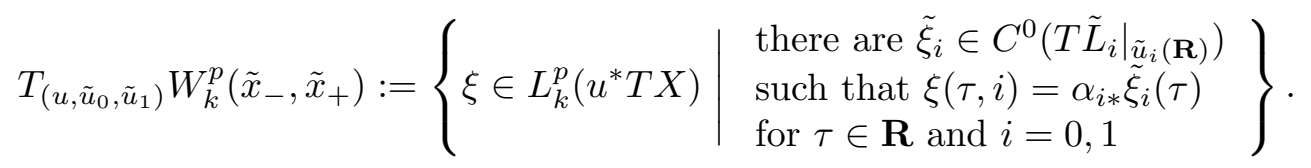

If $\xi \in T_{\left(u, \tilde{u}_{0}, \tilde{u}_{1}\right)} W_{k}^{p}\left(\tilde{x}_{-}, \tilde{x}_{+}\right)$, then $v(\tau, t):=\exp _{u(\tau, t)}^{g^{\left(t, s_{u}(\tau, t)\right)}} \xi(\tau, t)$ gives an element of $W_{k}^{p}\left(\tilde{x}_{-}, \tilde{x}_{+}\right)$. Then we obtain a similar theorem as in [4]:

Theorem 2.1. For $k p>2$ and $\tilde{x}_{-}, \tilde{x}_{+} \in I\left(\alpha_{0}, \alpha_{1}\right)$, the set $W_{k}^{p}\left(\tilde{x}_{-}, \tilde{x}_{+}\right)$is a smooth Banach manifold. The tangent space at $\left(u, \tilde{u}_{0}, \tilde{u}_{1}\right) \in W_{k}^{p}\left(\tilde{x}_{-}, \tilde{x}_{+}\right)$is given by $T_{\left(u, \tilde{u}_{0}, \tilde{u}_{1}\right)} W_{k}^{p}\left(\tilde{x}_{-}, \tilde{x}_{+}\right)$, and the exponential maps

$$
\exp _{u(\tau, t)}^{\left.g^{(t, s}(\tau, t)\right)}: T_{\left(u, \tilde{u}_{0}, \tilde{u}_{1}\right)} W_{k}^{p}\left(\tilde{x}_{-}, \tilde{x}_{+}\right) \rightarrow W_{k}^{p}\left(\tilde{x}_{-}, \tilde{x}_{+}\right)
$$

give smooth coordinate charts.

Given a family $J_{t}, t \in[0,1]$, of compatible almost complex structures with $\omega$, we can consider the following elliptic partial differential equation

$$
\bar{\partial}_{J_{t}} u(\tau, t):=\frac{\partial u(\tau, t)}{\partial \tau}+J_{t}(u(\tau, t)) \frac{\partial u(\tau, t)}{\partial t}=0
$$

on $W_{k}^{p}\left(\tilde{x}_{-}, \tilde{x}_{+}\right)$. Define the appropriate moduli space by

$$
\mathcal{M}_{J_{t} k}^{p}\left(\tilde{x}_{-}, \tilde{x}_{+}\right):=\left\{\left(u, \tilde{u}, \tilde{u}^{\prime}\right) \in W_{k}^{p}\left(\tilde{x}_{-}, \tilde{x}_{+}\right) \mid \bar{\partial}_{J_{t}} u(\tau, t)=0\right\} .
$$

Note that $\mathbf{R}$ acts on $\mathcal{M}_{J_{t} k}^{p}\left(\tilde{x}_{-}, \tilde{x}_{+}\right)$by $a \cdot u(\tau, t):=u(\tau+a, t)$ for $a \in \mathbf{R}$, and we denote the quotient by $\hat{\mathcal{M}}_{J_{t}}^{p}\left(\tilde{x}_{-}, \tilde{x}_{+}\right)$. Because the boundary of $\left(u, \tilde{u}_{0}, \tilde{u}_{1}\right) \in$ $W_{k}^{p}\left(\tilde{x}_{-}, \tilde{x}_{+}\right)$locally lies on Lagrangian submanifolds, we can use the elliptic regularity theorem not only for the interior points but also for the boundary points:

Proposition 2.2. For $k p>2$, if $\bar{\partial}_{J_{t}} u=0$ for $\left(u, \tilde{u}, \tilde{u}^{\prime}\right) \in W_{k}^{p}\left(\tilde{x}_{-}, \tilde{x}_{+}\right)$, then $u$ is smooth.

Hence we delete the subscripts $p$ and $k$ from $\mathcal{M}_{J_{t} k}^{p}\left(\tilde{x}_{-}, \tilde{x}_{+}\right)$. Moreover we can show the transversality as in [4], [12] and [15] by substituting our path space for the usual one:

Theorem 2.3. The operator $\bar{\partial}_{J_{t}}$ defines a smooth section of the smooth Banach space bundle $\mathcal{L}_{k-1}^{p}$ over $W_{k}^{p}\left(\tilde{x}_{-}, \tilde{x}_{+}\right)$whose fiber is $L_{k-1}^{p}\left(u^{*} T X\right)$, and so that $\mathcal{M}_{J_{t}}\left(\tilde{x}_{-}, \tilde{x}_{+}\right)$is the zero set of $\bar{\partial}_{J_{t}}$. The linearization

$$
E_{\left(u, \tilde{u}, \tilde{u}^{\prime}\right)}:=D \bar{\partial}_{J_{t}}: T_{\left(u, \tilde{u}, \tilde{u}^{\prime}\right)} W_{k}^{p}\left(\tilde{x}_{-}, \tilde{x}_{+}\right) \rightarrow L_{k-1}^{p}\left(u^{*} T X\right)
$$

with $k p>2$ is a Fredholm operator for $\left(u, \tilde{u}, \tilde{u}^{\prime}\right) \in \mathcal{M}_{J_{t}}\left(\tilde{x}_{-}, \tilde{x}_{+}\right)$. There is an open and dense set $\mathcal{J}_{\text {reg }}$ in $\mathcal{J}$, the set of smooth families $J_{t}$, such that, if $J_{t} \in \mathcal{J}_{\text {reg }}$, then $E_{\left(u, \tilde{u}, \tilde{u}^{\prime}\right)}$ is surjective for $\left(u, \tilde{u}, \tilde{u}^{\prime}\right) \in \mathcal{M}_{J_{t}}\left(\tilde{x}_{-}, \tilde{x}_{+}\right)$. 
Corollary 2.4. If $J_{t} \in \mathcal{J}_{\text {reg }}$, then $\mathcal{M}_{J_{t}}\left(\tilde{x}_{-}, \tilde{x}_{+}\right)$is a smooth finite dimensional manifold.

We can see the compactness of our moduli spaces. If we have a bubbling sequence, then we rescale the sequence and obtain a finite energy pseudoholomorphic map $\mathbf{C} \rightarrow X$ or $(\mathbf{H}, \mathbf{R}) \rightarrow\left(X, \alpha_{0}\left(\tilde{L}_{0}\right)\right.$ or $\left.\alpha_{1}\left(\tilde{L}_{1}\right)\right)$, where $\mathbf{H}$ is the upper half plane. The removal of singularities at interior points and smooth boundary points works [3] and [11], and also at self-intersection points by the removal of boundary singularity with boundary on Lagrangian immersions Theorem 1.4 in [8]. Moreover, the convergence to broken trajectories is standard [3]. Hence we obtain the following compactness theorem:

Theorem 2.5. Let $X$ be a closed symplectic manifold. Suppose $\int_{D^{2}} u^{*} \omega=0$ for $u:\left(D^{2}, \partial D^{2}\right) \rightarrow\left(X, \alpha_{0}\left(\tilde{L}_{0}\right)\right.$ or $\left.\alpha_{1}\left(\tilde{L}_{1}\right)\right)$. Then, for every finite number $c>0$, the subset of $\hat{\mathcal{M}}_{J_{t}}\left(\tilde{x}_{-}, \tilde{x}_{+}\right)$which consists of the elements satisfying $\int_{\mathbf{R} \times[0,1]} u^{*} \omega \leq c$ can be compactified (with respect to the topology of uniform convergence with all derivatives on compact set).

\section{Floer homology for Lagrangian immersions}

Let us denote by $P\left(\alpha_{0}, \alpha_{1}\right)$ the space of $\left(\delta, \delta_{0}, \delta_{1}\right)$, where $\delta:[0,1] \rightarrow X$ and $\delta_{i} \in \tilde{L}_{i}$ such that $\delta(i)=\alpha_{i}\left(\delta_{i}\right)$ for $i=0,1$. As in [2], we define admissible components of the path space. A component $P$ of $P\left(\alpha_{0}, \alpha_{1}\right)$ is called admissible if it satisfies the following two conditions $\mathcal{P}_{1}$ and $\mathcal{P}_{2}$ :

$\mathcal{P}_{1}$ For $\left(u, \tilde{u}_{0}, \tilde{u}_{1}\right) \in W^{\infty}\left(S^{1} \times[0,1], X ; \alpha_{0}, \alpha_{1}\right)$ such that $u\left(\tau_{0}, t\right) \in P$ for $\tau_{0} \in S^{1}, \int_{S^{1} \times[0,1]} u^{*} \omega=0$.

$\mathcal{P}_{2}$ For $\left(u, \tilde{u}_{0}, \tilde{u}_{1}\right) \in W^{\infty}\left(S^{1} \times[0,1], X ; \alpha_{0}, \alpha_{1}\right)$ such that $u\left(\tau_{0}, t\right) \in P$ for $\tau_{0} \in S^{1}$, the relative Maslov class $\mu\left(\left.u\right|_{S^{1} \times\{0\}},\left.u\right|_{S^{1} \times\{1\}}\right)$, see the definition in [2], vanishes.

The condition $\mathcal{P}_{1}$ implies the well-definedness of the symplectic action functional, and we need $\mathcal{P}_{2}$ to define the grading of Floer homology. There is an important case when admissible components exist:

Lemma 3.1. Let $\alpha_{0}=\alpha: \tilde{L} \rightarrow X$ and $\alpha_{1}=\varphi_{1} \circ \alpha: \tilde{L} \rightarrow X$.

(i) Suppose $\int_{D^{2}} u^{*} \omega=0$ for $u:\left(D^{2}, \partial D^{2}\right) \rightarrow(X, \alpha(\tilde{L}))$. Then the components which contain constant paths in $\alpha_{0}(\tilde{L}) \cap \alpha_{1}(\tilde{L})$ satisfy $\mathcal{P}_{1}$.

(ii) Suppose the boundary homomorphism $\pi_{2}(X, \alpha(\tilde{L})) \rightarrow \pi_{1}(\alpha(\tilde{L}))$ is trivial. Then the components as in $(i)$ satisfy $\mathcal{P}_{2}$.

Note that every intersection point determines a constant path and these constant paths may lie in different path components for different intersection points. We will define Floer homology of the triple $\alpha_{0}, \alpha_{1}$ and $P$.

Denote by $I\left(\alpha_{0}, \alpha_{1}, P\right)$ the subset of $I\left(\alpha_{0}, \alpha_{1}\right)$ which consists of those intersection points which belong to $P$. From $\mathcal{P}_{2}$, there exists a map $\mu: I\left(\alpha_{0}, \alpha_{1}, P\right) \rightarrow \mathbf{Z}$ as usual such that the index of $E_{\left(u, \tilde{u}, \tilde{u}^{\prime}\right)}$ is $\mu\left(\tilde{x}_{-}\right)-\mu\left(\tilde{x}_{+}\right)$. For $J_{t} \in \mathcal{J}_{\text {reg }}$, by the 
compactness theorem, we can count the number of the elements of $\hat{\mathcal{M}}_{J_{t}}\left(\tilde{x}_{-}, \tilde{x}_{+}\right)$ modulo 2 when $\mu\left(\tilde{x}_{-}\right)-\mu\left(\tilde{x}_{+}\right)=1$. We define the chain complex by

$$
C F_{k}=C F_{k}\left(\alpha_{0}, \alpha_{1}, P\right):=\bigoplus_{\tilde{x} \in I\left(\alpha_{0}, \alpha_{1}, P\right), \mu(\tilde{x})=k} \mathbf{Z}_{2} \tilde{x}
$$

with $\partial: C F_{k} \rightarrow C F_{k-1}$ in terms of the canonical bases

$$
\partial \tilde{x}_{-}:=\sum_{\mu\left(\tilde{x}_{+}\right)=k-1} \sharp \hat{\mathcal{M}}_{J_{t}}\left(\tilde{x}_{-}, \tilde{x}_{+}\right) \cdot \tilde{x}_{+} .
$$

From the surjectivity in Theorem 2.3 the gluing as in [3] carry over to our situation, and then from the compactness we obtain the following proposition:

Proposition 3.2. Suppose the same assumptions as in Theorem 2.5. If $J_{t} \in$ $\mathcal{J}_{\text {reg }}$, then $\partial \circ \partial=0$.

We call the homology of $\left(C F_{*}, \partial\right)$ Floer homology for Lagrangian immersions and denote it by $H F\left(\alpha_{0}, \alpha_{1}, P ; J_{t}\right)$. Although the definition of $H F\left(\alpha_{0}, \alpha_{1}, P ; J_{t}\right)$ depends on $J_{t}, H F\left(\alpha_{0}, \alpha_{1}, P ; J_{t}\right)$ and $H F\left(\alpha_{0}, \alpha_{1}, P ; J_{t}^{\prime}\right)$ are isomorphic when $J_{t}$ and $J_{t}^{\prime} \in \mathcal{J}_{\text {reg }}$, and moreover there exists an isomorphism $H F_{*}\left(\alpha_{0}, \alpha_{1}, P ; J_{t}\right) \rightarrow$ $H F_{*}\left(\varphi_{0}^{-1} \circ \alpha_{0}, \varphi_{1}^{-1} \alpha_{1}, \varphi^{*} P ; J_{t}\right)$, where $\varphi^{*} P$ denotes the component of the path $\varphi_{t}^{-1}(\delta(t))$ for $\delta(t) \in P$, see [2]. From these, we can drop $J_{t}$ from the notation of Floer homology and $H F_{*}\left(\alpha_{0}, \alpha_{1}, P\right)$ is invariant under Hamiltonian isotopy of $\alpha_{1}\left(\tilde{L}_{1}\right)$.

Note that, for different admissible components $P_{1}$ and $P_{2}$, we can not canonically determine the difference between the gradings of the Floer homologies. Nonetheless we can define $H F_{*}\left(\alpha_{0}, \alpha_{1}, P_{1} \sqcup P_{2}\right)$ which is isomorphic to $H F_{*}\left(\alpha_{0}, \alpha_{1}, P_{1}\right) \oplus H F_{*}\left(\alpha_{0}, \alpha_{1}, P_{2}\right)$ up to the difference between the gradings of the two components.

\section{Calculation of Floer homology}

Let $\alpha: \tilde{L} \rightarrow X$ be a Lagrangian immersion as in Theorem 1.1. The components of $P\left(\alpha_{0}, \alpha_{1}\right)$ which contain constant paths in $\alpha(\tilde{L}) \cap\left(\varphi_{1} \circ \alpha\right)(\tilde{L})$ are admissible. Hence we denote by $\mathcal{P}$ the union of the admissible components. Since the Floer homology $H F_{*}\left(\alpha, \varphi_{1} \circ \alpha, \mathcal{P}\right)$ is independent of Hamiltonian isotopies, we will calculate it for convenient Hamiltonian functions.

Each point $p$ of $\tilde{L}$ has a neighborhood $U_{p}$ such that $\alpha\left(U_{p}\right)$ is a submanifold of $X$. We take a tubular neighborhood of $\alpha\left(U_{p}\right)$ with a small radius and take a diffeomorphism $f_{p}$ from $U_{p} \times B$ to the tubular neighborhood, where $B$ is an $n$-dimensional ball. If $x \in U_{p} \times B$ and $y \in U_{q} \times B$ satisfy $f_{p}(x)=f_{q}(y)$, then we introduce an equivalence relation $x \sim y$ to define a manifold $V_{\tilde{L}}:=$ $\sqcup_{p \in \tilde{L}}\left(U_{p} \times B\right) / \sim$, and identify $\tilde{L}$ with $\tilde{L} \times\{0\} \subset V_{\tilde{L}}$. Moreover we also define a map $f: V_{\tilde{L}} \rightarrow X$ which locally coincides with the diffeomorphisms $f_{p}$. Then the manifold $V_{\tilde{L}}$ with $f^{*} \omega$ is symplectically isomorphic to a neighborhood of the zero section $(\tilde{L})_{0}$ of the cotangent bundle $T^{*} \tilde{L}$ with the canonical symplectic structure. 
For Lagrangian submanifolds, the following proposition was implicitly used by Floer [1], and the proof for immersions is completely similar to that for embeddings [1] and [14]:

Proposition 4.1. There exists a constant $\kappa$ which satisfies the following: for $0<\delta<\kappa$ there is a constant $c_{\delta}$ such that, if the $C^{1}$-norm of a Hamiltonian function is smaller than $c_{\delta}$, then $\operatorname{dist}(u(\tau, 0), u(\tau, 1))<\delta$ for $\left(u, \tilde{u}, \tilde{u}^{\prime}\right) \in$ $\mathcal{M}_{J_{t}}\left(\tilde{x}_{-}, \tilde{x}_{+}\right)$. [10]:

To prove this proposition, we refer to the following lemma in Chapter 4 of

Lemma 4.2. There exists a constant $\hbar>0$ such that the following holds: if $r>0$ such that $D_{r}\left(z_{0}\right):=\left\{z \in \mathbf{C}|| z-z_{0} \mid<r\right\} \subset \mathbf{R} \times(0,1)$ and $u$ : $D_{r}\left(z_{0}\right) \rightarrow X$ is a solution of $\bar{\partial}_{J_{t}} u=0$ such that $\int_{D_{r}\left(z_{0}\right)}|d u|^{2}<\hbar$, then $\left|d u\left(z_{0}\right)\right|^{2} \leq \frac{8}{\pi r^{2}} \int_{D_{r}\left(z_{0}\right)}|d u|^{2}$.

Proof of Proposition 4.1. To emphasize Hamiltonian isotopy $\varphi:=\left\{\varphi_{t}\right\}_{0 \leq t \leq 1}$ we shall use notation $\mathcal{M}_{J_{t}}^{\varphi}:=\bigcup \mathcal{M}_{J_{t}}\left(\tilde{x}_{-}, \tilde{x}_{+}\right)$, where the union ranges over $I\left(\alpha, \varphi_{1} \circ \alpha\right)$, and moreover, for $0<c<\infty$, we put $\mathcal{M}_{J_{t}}^{c}:=\bigcup_{\varphi} \mathcal{M}_{J_{t}}^{\varphi}$, where the union ranges over the Hamiltonian isotopies such that the symplectic area of every element of $\mathcal{M}_{J_{t}}^{\varphi}$ is smaller than $c$. Then there is a constant $C$, which is independent of $\left(u, \tilde{u}, \tilde{u}^{\prime}\right) \in \mathcal{M}_{J_{t}}^{c}$, such that $|d u(z)| \leq C, z \in \mathbf{R} \times[0,1]$; since, if there is no such constant, we can choose sequences $\left\{\left(u_{i}, \tilde{u}_{i}, \tilde{u}_{i}^{\prime}\right)\right\} \subset \mathcal{M}_{J_{t}}^{c}$ and $\left\{z_{i}\right\} \subset \mathbf{R} \times[0,1]$ such that $\lim _{i \rightarrow \infty}\left|d u_{i}\left(z_{i}\right)\right|=\infty$ from which we can rescale a bubbling sequence, which contradicts to $\pi_{2}(X, \alpha(\tilde{L}))=0$.

Denote $\kappa:=\min \left\{1 / 2, c^{1 / 4}, \hbar^{1 / 4}\right\}$. For $0<\delta<\kappa$, we put $r=\delta$ in Lemma 4.2, then the following inequality holds: for $\left(u, \tilde{u}, \tilde{u}^{\prime}\right) \in \mathcal{M}_{J_{t}}^{\delta^{4}}$ and $z_{0} \in \mathbf{R} \times[\delta, 1-\delta]$

$$
\left|d u\left(z_{0}\right)\right|^{2} \leq \frac{8}{\pi \delta^{2}} \int_{D_{\delta}\left(z_{0}\right)}|d u|^{2} \leq \frac{8}{\pi \delta^{2}} \delta^{4}
$$

On the other hand we can take a constant $c_{\delta}$ such that, if $C^{1}$-norm of Hamiltonian function is smaller than $c_{\delta}$, then the symplectic area of every element of $\mathcal{M}_{J_{t}}^{\varphi}$ is smaller than $\delta^{4}$, which is possible from the well-defindness of the symplectic action functional.

From these, if the $C^{1}$-norm of the Hamiltonian function is smaller than $c_{\delta}$, then for $\left(u, \tilde{u}, \tilde{u}^{\prime}\right) \in \mathcal{M}_{J_{t}}^{\varphi}$

$$
\begin{aligned}
\operatorname{dist}(u(\tau, 0), u(\tau, 1)) & \leq \int_{0}^{1} \sqrt{g\left(\frac{\partial u}{\partial t}, \frac{\partial u}{\partial t}\right)} d t \\
& \leq G_{1}\left(\int_{t \in[0, \delta] \cup[1-\delta, 1]}\left|\frac{\partial u}{\partial t}\right| d t+\int_{t \in[\delta, 1-\delta]}\left|\frac{\partial u}{\partial t}\right| d t\right) \\
& \leq G_{2} \delta
\end{aligned}
$$

where $G_{1}$ and $G_{2}$ are constants depending only on $g$. 
Proof of Theorem 1.1. We denote the image of $\alpha$ by $L$ and denote the transverse self-intersection points of $L$ by $p_{1}, \ldots, p_{N} \in L$. Here we take closed $2 n$ dimensional balls $B_{\varepsilon}\left(p_{j}\right)$ in $X$, centered at $p_{j}$ of small radius $\varepsilon$, such that they do not intersect each other and $B_{\varepsilon}\left(p_{j}\right) \cap L$ are homeomorphic to two $n$-disks $L_{p_{j} 1}$ and $L_{p_{j} 2}$ intersecting at $p_{j}$. Moreover we also take $\varepsilon$ small enough such that $L_{p_{j} 1}$ and $L_{p_{j} 2}$ look approximately like linear planes. Next we consider the following maps $u$ : there is $\rho_{j}>0$ and $\xi \in L_{k}^{p}\left(T_{p_{j}} X\right)$ such that $u(\tau, t)=\exp _{p_{j}} \xi(\tau, t)$ for $\tau>\rho_{j}$, and moreover $u(\tau, 0): \mathbf{R} \rightarrow L_{p_{j} 1}$ and $u(\tau, 1): \mathbf{R} \rightarrow L_{p_{j} 2}$ for large $\tau$, and $u(\mathbf{R}, 0) \cap\left(X \backslash B_{\varepsilon / 2}\left(p_{j}\right)\right) \neq \emptyset$ and $u(\mathbf{R}, 1) \cap\left(X \backslash B_{\varepsilon / 2}\left(p_{j}\right)\right) \neq \emptyset$. Then we put

$$
w(\alpha):=\min _{p_{j}} \inf _{u} \max _{\tau \in \mathbf{R}} \operatorname{dist}(u(\tau, 0), u(\tau, 1)),
$$

where $u$ satisfies the above conditions. Because the self-intersection points of $\alpha$ are transverse, this quantity $w(\alpha)$ is greater than zero. First we choose $\delta$ such that $\delta<\min \{w(\alpha), \kappa\}$ and the $\delta$-neighborhood $U_{\delta}$ of $\alpha(\tilde{L})$ contained in $f\left(V_{\tilde{L}}\right)$, and moreover we choose a Hamiltonian function $H$ such that the $C^{1}$ norm is smaller than $c_{\delta}$ and, in $B_{\varepsilon}\left(p_{j}\right)$, the Hamiltonian isotopy associated with $H$ looks like a parallel translation $P_{p_{j}}$ such that $L_{p_{j} h}$ and $P_{p_{j}}\left(L_{p_{j} k}\right), h \neq k$, intersect transversally at one point, and $L_{p_{j} h}$ and $P_{p_{j}}\left(L_{p_{j} h}\right), h=1,2$, do not intersect. Note that the number of the intersection points of $L$ and $\varphi_{1}(L)$ in $B_{\varepsilon}\left(p_{j}\right)$ is two. Then there are two kinds of intersection points of $L$ and $\varphi_{1}(L)$, i.e., the intersection points look like $L_{p_{j} h} \cap P_{p_{j}}\left(L_{p_{j} k}\right)$ in $B_{\varepsilon}\left(p_{j}\right)$ and the others (away from the balls). From the definition of $w(\alpha)$ and Proposition 4.1, there is no element of $\mathcal{M}_{J_{t}}(x, y)$, where $x$ or $y$ is of the type $L_{p_{j} h} \cap P_{p_{j}}\left(L_{p_{j} k}\right)$. Hence $\left(C F_{*}\left(\alpha, \varphi_{1} \circ \alpha, \mathcal{P}\right), \partial\right)$ turns out to be the following form

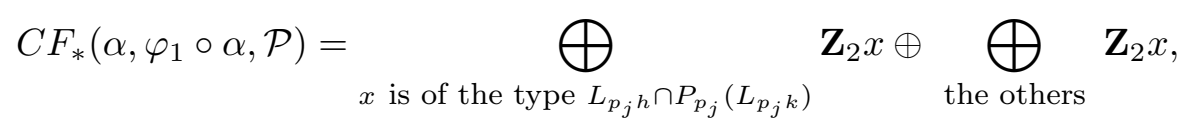

and the boundary operator $\partial$ splits as well as the above splitting of $C F_{*}$ such that $\partial$ is trivial on the first component, i.e., $\partial=0 \oplus \partial_{2}$. Note that the admissible component for the intersections of the other type may or may not contain intersection points of the type $L_{p_{j} h} \cap P_{p_{j}}\left(L_{p_{j} k}\right)$, and that we omit the grading since we do not use it here.

Let $\left\{\tilde{\varphi}_{t}\right\}_{0 \leq t \leq 1}$ be a Hamiltonian isotopy on $T^{*} \tilde{L}$ associated with a Hamiltonian function $\tilde{H}: T^{*} \tilde{L} \rightarrow \mathbf{R}$ which coincides with $H \circ f$ on $V_{\tilde{L}}$. Then the set of the intersection points of $(\tilde{L})_{0}$ and $\tilde{\varphi}_{1}\left((\tilde{L})_{0}\right)$ coincides with the subset consisting of the others in $I\left(\alpha, \varphi_{1} \circ \alpha\right)$. A similar argument of Proposition 4.1 holds for $(\tilde{L})_{0}$ and $\tilde{\varphi}_{1}\left((\tilde{L})_{0}\right) \subset T^{*} \tilde{L}$, and then, from the boundary condition of the path spaces, the moduli spaces $\mathcal{M}_{J_{t}}(x, y)$, where $x$ and $y$ are of the other type, coincide with the usual moduli spaces of pseudo-holomorphic strips for $x, y \in(\tilde{L})_{0} \cap \tilde{\varphi}_{1}\left((\tilde{L})_{0}\right)$. Then, we find that the chain complex $\left(\bigoplus_{\text {the others }} \mathbf{Z}_{2} x, \partial_{2}\right)$ is nothing but Floer's chain complex for $(\tilde{L})_{0}$ and $\tilde{\varphi}_{1}\left((\tilde{L})_{0}\right)$ whose homology group is isomorphic to the singular homology $H_{*}\left(\tilde{L}, \mathbf{Z}_{2}\right)$ of $\tilde{L}$, see [3] and [5]. Then Floer homology 
groups $H F_{*}\left(\alpha, \varphi_{1} \circ \alpha, \mathcal{P}\right)$ is isomorphic to $\bigoplus^{2 N} \mathbf{Z}_{2} \oplus H_{*}\left(\tilde{L}, \mathbf{Z}_{2}\right)$. We finish proving Theorem 1.1.

Acknowledgment: The author would like to thank K. Fukaya for many useful discussions and great encouragement.

\section{References}

[1] Y.V. Chekanov, Hofer's symplectic energy and Lagrangian intersections, in "Contact and Symplectic Geometry (Cambridge, 1994)", 296-306, Publ. Newton Inst., 8, Cambridge Univ. Press, Cambridge, 1996.

[2] Y. Eliashberg, H. Hofer and D. Salamon. Lagrangian intersections in contact geometry, Geom. Funct. Anal. 5, No. 2, (1995) 245-269.

[3] A. Floer, Morse theory for Lagrangian intersections, J. Differential Geom. 28, No. 3, 513-547 (1988).

[4] - The Unregularized Gradient Flow of the Symplectic Action, Comm. Pure. Appl. Math. 41, 775-813 (1988).

[5] W Witten's complex and infinite dimensional Morse theory, J. Differential Geom. 30, No.1, 207-221 (1989).

[6] A. Floer, H. Hofer and D. Salamon. Transversality in elliptic Morse theory for symplectic action, Duke Math. J. 80 (1995) 251-292.

[7] M. Gromov, Pseudo-holomorphic curves in symplectic manifolds, Invent. Math. 82 (1985) 307-347.

[8] S. Ivashkovich and V. Shevchishin, Reflection principle and J-complex curves with boundary on totally real immersions, Commun. Contemp. Math. 4, No. 1, (2002) 65-106.

[9] D. Kwon and Y.-G. Oh, Structure of the image of (pseudo)-holomorphic discs with totally real boundary condition. Appendix 1 by J. P. Rosay, Comm. Anal. Geom. 8, No.1, (2000) 31-82.

[10] D. McDuff and D. Salamon, J-holomophic curves and quantum cohomology, Colloquium Pub., 52, Amer. Math. Soc., Providence, RI, 2004.

[11] Y.-G. Oh, Removal of boundary singularities of pseudo-holomorphic curves with Lagrangian boundary conditions, Comm. Pure Appl. Math. 45 (1992) 121-139.

[12] _ Floer cohomology of Lagrangian intersections and pseudo-holomorphic disks I, Comm. Pure Appl. Math. 46 (1993) 949-993.

[13] Addendum to "Floer cohomology of Lagrangian intersections and pseudoholomorphic disks I", Comm. Pure Appl. Math. 48 (1995) 1299-1302.

[14] Floer cohomology, spectral sequences and the Maslov class of Lagrangian embeddings in $\mathbf{C}^{n}$, Internat. Math. Res. Notices no. 7 (1996) 305-346.

[15] On the structure of pseudo-holomorphic discs with totally real boundary conditions, J. Geom. Anal. 7 (1997) 305-327.

Department of Mathematics, Tokyo Metropolitan University

E-mail address: akaho@math.metro-u.ac.jp 